\title{
SÍNDROME DE OVARIO POLIQUÍSTICO, ENDOMETRIO Y RIESGO DE ABORTO
}

\section{Polycystic ovarian syndrome, endometrium and abortion risk}

Carlos Arturo Vivas, M.D.*

Recibido: septiembre 2/2005 - Revisado: octubre 11/2005 - Aceptado: noviembre 11/2005

\section{RESUMEN}

El síndrome de ovario poliquístico (SOP) es la endocrinopatía más frecuente en mujeres en edad reproductiva, se observa en el 5-7\% de ellas. El diagnóstico se hace ante la presencia de por lo menos dos componentes de la triada: oligoovulación, hiperandrogenismo y ovarios poliquísticos. Estas mujeres tienen niveles elevados de lutropina (LH), hiperinsulinemia e hiperandrogenismo y se ha reportado un aumento en la incidencia de aborto en este grupo de pacientes. Este hecho podría estar relacionado con los cambios endometriales observados en estas mujeres y que se asocian a niveles elevados de andrógenos e insulina, provocando así, inadecuada implantación y probablemente mayor pérdida del embarazo en el primer trimestre. Medidas terapéuticas, como el uso de sensibilizantes a la acción de la insulina, que contribuyen a disminuir los niveles de insulina y por lo tanto de andrógenos, podrían tener un efecto favorable al disminuir la incidencia de aborto en pacientes con SOP.

Palabras clave: síndrome de ovario poliquístico, aborto, endometrio, insulina

\section{SUMMARY}

Polycystic ovarian syndrome is the most frequent endocrinopathy in reproductive age women (5-7\%).

\footnotetext{
* Especialista en Ginecología y Obstetricia. Universidad Javeriana, Profesor de la Universidad del Tolima, Calle 47 No 4 - 26, Centro Médico Javeriano. Cons. 407, Correo electrónico: unifertil@andinet.com
}

The diagnosis is made when two out of three components are present, such as hyperandrogenism, polycystic ovaries and oligoovulation. These women have high levels of luteinizing hormone (LH), hyperisulinemia and hyperandrogenism and an increased rate of abortion has been reported in these patients. This fact could be related to changes found in the endometrium and the elevated serum levels of androgens and insulin. These changes can lead to an inadequate implantation and probably to an increase in first trimester pregnancy losses.

Therapeutic approaches such as the use of insulin-sensitizing drugs to decrease insulin levels could be useful in decreasing abortion rates in these patients.

Key words: polycystic ovarian syndrome, endometrium, abortion, insulin.

\section{INTRODUCCIÓN}

El 5-7\% de las pacientes en edad reproductiva padecen el síndrome de ovario poliquístico (SOP). Desde la publicación del trabajo de Stein y Leventhal en 1935, quienes describieron 7 mujeres con amenorrea, hirsutismo, obesidad y ovarios de apariencia poliquística, el entendimiento de esta enfermedad ha cambiado de manera importante. Hoy en día, es ampliamente aceptado que el SOP es una condición asociada a una resistencia aumentada a la acción de la insulina, que produce hiperinsulinemia y, como consecuencia, una secreción anormal de andrógenos 
ováricos y gonadotropinas. Estos cambios endocrinos se reflejan en desordenes en el ciclo menstrual, anovulación y exceso de andrógenos. Se reconoce también hoy que esta condición tiene grandes implicaciones, tanto en la vida reproductiva, como en el metabolismo y la salud cardiovascular presente y futura de la mujer. ${ }^{1-3}$

Se ha reportado un aumento en la incidencia de aborto (entre el 30-50\%, 3 veces la incidencia en la población general) en este grupo de pacientes. ${ }^{4}$ Buscar las causas de esta asociación es el propósito de esta revisión.

Se realizará una mirada a los conceptos fisiopatológicos básicos del SOP, su posible relación con los cambios endometriales y el riesgo de aborto.

\section{Fisiopatología del síndrome de ovario poliquístico}

Las sociedades europea y estadounidense de medicina reproductiva se pronunciaron recientemente llegando a un consenso para el diagnóstico y definición del síndrome de ovario poliquístico. Se definieron como criterios diagnósticos los siguientes: $:^{2,5}$

1. Oligo o anovulación;

2. Signos clínicos y/o bioquímicos de hiperandrogenismo;

3. Al menos un ovario con 12 o más folículos entre 2-9 $\mathrm{mm}$ y/o un volumen mayor de $10 \mathrm{ml}$.

Dos de los tres criterios deben estar presentes para el diagnóstico y otros desordenes endocrinos deben ser excluidos (por ejemplo, enfermedad tiroidea, hiperplasia suprarrenal congénita, hiperprolactinemia, tumor secretor de andrógenos, síndrome de Cushing, entre otros). El consenso no recomienda medir resistencia a la insulina, aunque muchos autores recomiendan realizar prueba de tolerancia a la glucosa en este grupo de pacientes. ${ }^{2}$

Recordemos que la LH estimula la producción de andrógenos en las células de la teca y la folitropina (FSH) estimula la producción de estrógenos a partir de los andrógenos en las células de la granulosa.
La biosíntesis de los andrógenos es mediada por el Citocromo P-450c17, una enzima con actividad 17 $\alpha$ - hidroxilasa y 17,20 -liasa, que son requeridas para la formación de androstenediona, que a su vez es convertida en testosterona por la $17 ß$ hidroxiesteroidedeshidrogenasa (17ßHSD) o aromatizada por la aromatasa (P-450arom) a estrona. ${ }^{6}$

En el SOP se observa una concentración proporcionalmente elevada de LH con respecto a la de FSH, por lo que los ovarios de estas mujeres sintetizan preferiblemente andrógenos. Como la secreción de gonadotropinas depende de los cambios en la frecuencia y amplitud de los pulsos de gonadoliberina $(\mathrm{GnRH})$, se sugiere que un incremento en la frecuencia de los pulsos de GnRH explicaría el aumento de la secreción de LH. Se sugiere además, que esta frecuencia acelerada de los pulsos puede ser secundaria a los bajos niveles de progesterona resultantes de la oligoovulación de estas pacientes. ${ }^{6}$ En 1970 Yen y cols. ${ }^{7}$ propusieron estas alteraciones como el fenómeno fisiopatológico básico en el SOP al encontrar niveles elevados de LH, asociados con un incremento en la frecuencia y amplitud de sus pulsos. ${ }^{1,3}$

Posteriormente, Kenneth Ryan en 1983, sugirió una relación entre la hiperinsulinemia y el hiperandrogenismo, asociado con un aumento de la resistencia a la insulina. Estudios in vivo e in vitro han sugerido que las células de la teca de las mujeres con SOP, producen una mayor cantidad de testosterona que las de mujeres normales, reforzando la idea de una relación causa efecto entre la resistencia a la insulina, el hiperandrogenismo y la anovulación. ${ }^{1,6,8-10}$

La insulina ha mostrado un papel directo e indirecto en la hiperandrogenemia de las mujeres con SOP; de manera directa, aumentando la producción de andrógenos por las células de la teca, potenciando la acción de la LH sobre estas células, y de manera indirecta inhibiendo la síntesis de la globulina ligadora de hormonas sexuales (SHGB), incrementando la proporción de testosterona libre que es biológicamente activa. 
En síntesis una mujer con SOP tiene niveles elevados de LH, hiperinsulinemia e hiperandrogenismo. La influencia de estos cambios hormonales sobre la adecuada implantación y placentación será objeto de análisis posterior.

\section{El endometrio en el SOP}

Los cambios fisiológicos del endometrio en un ciclo ovulatorio se pueden resumir de la siguiente manera: inicialmente los niveles de estrógenos se elevan progresivamente produciendo un estímulo en la proliferación endometrial, que se refleja en su crecimiento. Después de la ovulación y como consecuencia de los niveles circulantes de estradiol y progesterona, se inicia la fase secretora, en ella es importante el efecto de la progesterona sobre la síntesis del receptor de estradiol, inhibiendo la proliferación, disminuyendo la mitosis y la síntesis de ADN. Siete a diez días después de la ovulación y bajo la influencia de la progesterona el endometrio se vuelve receptivo para la implantación (ventana de implantación). Las células predeciduales, que aparecen hacia la mitad de la fase secretora, cumplen un importante papel al secretar una serie de factores de acción paracrina (prolactina, IGF, IGFBP, relaxina y otros) que actúan sobre la receptividad endometrial, la adhesión embrionaria y la invasión trofoblástica.

Cuando no hay ovulación los efectos de la progesterona no se producen, no sobreviene la transformación secretora y el endometrio es continuamente expuesto al efecto mitogénico de los estrógenos, produciéndose un crecimiento anormal del endometrio y patrones impredecibles de sangrado.

La normalidad de estos cambios endometriales se ve reflejada en la aparición sincrónica de una serie de elementos que se han estudiado de manera extensa y que pueden predecir el grado de receptividad endometrial. Algunos de estos marcadores de receptividad endometrial han mostrado en pacientes con SOP diferencias con el patrón normal. A continuación mencionaremos algunas de ellas.
Receptores esteroideos: los niveles de los receptores de andrógenos (RA), los receptores de estrógenos (RE) y los receptores de progesterona (RP) cambian de acuerdo con el momento del ciclo menstrual normal. ${ }^{2}$

- Los RA se observan disminuidos desde la fase folicular temprana hasta la fase lútea media.

- $\operatorname{Los} R E(\alpha$ y $\beta)$ tienen su más alta concentración en la fase proliferativa tardía y disminuyen después de la ovulación.

- Los RP (A y B) tienen su pico en el momento de la ovulación.

A diferencia de estos hallazgos normales, en las mujeres con SOP se encuentran niveles elevados de RA y un aumento de los RE $(\alpha)$ en la fase lútea media. $^{2}$

\section{Marcadores de receptividad endometrial:}

Dentro del proceso fisiológico de la implantación se han identificado una serie de elementos tales como, la aparición de los pinópodos, el aumento de la glicodelina A, presencia de moléculas de adhesión (mucinas, integrinas, tropininas), citocinas y factores de crecimiento (factor de crecimiento epidérmico, factor estimulante de colonias-1, factor inhibidor de la leucemia, interleucina 1, IGFBP, factor de crecimiento transformador $\beta$ ), y genes HOXA, que han sido asociados con una adecuada receptividad endometrial y al éxito de la implantación. ${ }^{11,12}$

Algunos de estos marcadores también han sido estudiados en mujeres con SOP:

- Las integrinas, son moléculas de adhesión y la expresión de sus unidades $\propto$ y $\beta$ es asociada a la receptividad endometrial. La aparición de la integrina $\alpha v \beta 3$ el día 19 del ciclo corresponde con la implantación del blastocisto. ${ }^{12}$ Se ha encontrado ausencia o aparición tardía de la integrina $\alpha v \beta 3$ en el SOP. ${ }^{2}$

- La glicodelina A, que es una glicoproteína con propiedades inmunosupresoras, que aumenta en la fase secretora, ${ }^{12}$ se encuentra disminuida en pacientes con SOP, por acción de los andrógenos, que inhiben su secreción. ${ }^{2}$ 
Estos cambios podrían relacionarse con una baja tasa de implantación o una alta incidencia de aborto.

\section{Insulina, factor de crecimiento similar a la insulina (IGF) y sus receptores en el endometrio: como ya se mencionó, los niveles} elevados de insulina cumplen un papel importante en la fisiopatología del SOP. La insulina actúa en el endometrio a través de su propio receptor, y en altas concentraciones, a través del receptor de IGF.

El factor de crecimiento similar a la insulina-1 (IGF-1), el factor de crecimiento similar a la insulina-2 (IGF-2) y la proteína ligadora del factor de crecimiento similar a la insulina - 1 (IGFBP-1), son importantes en los cambios de proliferación, desarrollo e implantación a nivel del endometrio. El IGF-1 se correlaciona con los niveles de estradiol y participa como mediador de sus efectos; en cambio el IGF-2 es producido en la fase secretora y es regulado por la progesterona, su expresión en el citotrofoblasto le atribuye un importante papel en la invasión al endometrio. ${ }^{11}$ La IGFBP-1, es producida por el estroma endometrial decidualizado y regula los IGF, disminuyendo sus niveles libres. Su papel es fundamental en el anclaje del embrión y limita la invasión trofoblástica. Bajos niveles de IGFBP-1 se asocian a niveles altos de insulina. ${ }^{2,11}$

En las pacientes con SOP, tanto la insulina como el IGF, inhiben la decidualización endometrial. Estudios in vitro sugieren que la insulina interfiere con la decidualización a través de un efecto sobre las células estromales endometriales. ${ }^{2}$ Algunos autores sostienen que la hiperinsulinemia también tiene efectos adversos sobre la función endometrial afectando la ventana de implantación por disminución en la expresión de la glicodelina y la IGFBP-1. ${ }^{13}$

En resumen las pacientes con SOP tienen cambios endometriales que se reflejan en: a) una alteración del patrón de aparición y concentración de los receptores de hormonas esteroideas, b) anormalidad en la aparición de los marcadores de receptividad endometrial y c) decidualización anómala como consecuencia de los niveles elevados de insulina y los IGF. Se podría especular que como consecuencia clínica de estos cambios se observa, no solo una disminución de la tasa de embarazo, sino también una implantación anormal y un aumento en la incidencia de aborto.

\section{ABORTO Y SOP}

Se ha reportado una incidencia de aborto entre el 30-50\% de las pacientes con SOP que se embarazan. ${ }^{4}$ A su vez, el 30\% de las pacientes abortadoras habituales tienen SOP. Hallazgos frecuentes en estas pacientes con pérdidas recurrentes, tales como, niveles elevados de LH, aumento de la testosterona libre, niveles bajos de progesterona en fase lútea y desarrollo endometrial tardío, son similares a aquellos observados en pacientes con ovarios poliquísticos.

Se han hecho estudios que sugieren que la hipersecreción de LH en las mujeres con SOP podría predisponer al aborto después de la concepción espontánea o asistida. ${ }^{14-16}$

Otros factores de riesgo para pérdida temprana del embarazo en el SOP son la obesidad y la elevación sérica de los niveles de andrógenos. La obesidad se caracteriza por un aumento de la resistencia a la insulina con una hiperinsulinemia compensatoria. En un reciente estudio se correlacionó la hiperinsulinemia como un factor de riesgo independiente para la pérdida del embarazo. ${ }^{17}$

La actividad del inhibidor del activador del plasminógeno plasmático 1 (PAI-1) es mayor en mujeres con SOP. Aunque la actividad hipofibrinolítica del PAI es un factor de riesgo independiente para aborto, también se ha asociado con un aumento de la resistencia a la insulina. ${ }^{18,19}$

No se podría dejar de mencionar que la receptividad endometrial no es el único determinante para la exitosa implantación, otros factores, como calidad del ovocito y del embrión influyen directamente en la adecuada implantación y producen confusión al realizar estos análisis. 


\section{OPCIONES TERAPÉUTICAS}

El uso de sensibilizantes a la acción de la insulina, especialmente la metformina (medicamento categoría B para su uso en el embarazo), ${ }^{20}$ ha mostrado disminución en la tasa de abortos ${ }^{4,21,22}$ en las mujeres con SOP.

La metformina es una biguanida que actúa mejorando la sensibilidad a la insulina en el tejido periférico (músculo esquelético) y en el hígado, disminuye la producción basal de glucosa hepática, reduce los niveles de insulina, LH y testosterona, y aumenta los niveles de globulina ligadora de hormonas esteroideas. ${ }^{20}$

La terapia con metformina en pacientes con SOP ha mejorado la tasa de embarazo en ciclos estimulados con clomifeno. En pacientes con niveles elevados de insulina y clomifeno resistentes tratadas con metformina, llevadas a fertilización in vitro (FIV), se observó un mayor número de ovocitos maduros aspirados y una mayor tasa de fertilización y de embarazo. ${ }^{23}$

Algunos estudios sugieren una disminución del riesgo de aborto con el uso de metformina durante el primer trimestre de embarazo en pacientes con SOP. ${ }^{4,20}$ Un estudio controlado con placebo evidenció niveles superiores de glicodelina y de IGFBP en el grupo que tomó metformina. ${ }^{22}$ La terapia con metformina disminuyó la actividad del PAI-1 sin disminuir los niveles de glucosa plasmática en mujeres euglucémicas con SOP y esto podría por tánto, reducir la frecuencia de aborto espontáneo en el primer trimestre. ${ }^{4}$ Esta acción de la metformina de reducir la actividad del PAI-1 esta relacionada con la reducción sérica de los niveles de insulina. ${ }^{19}$

Varios reportes del doctor Glueck, en hijos nacidos de mujeres tratadas con metformina durante el embarazo no han mostrado efectos teratogénicos, ni alteraciones en el desarrollo durante los primeros 6 meses de vida. ${ }^{24,25}$

A su vez es claro que otros sensibilizantes a la acción de la insulina tales como la rosiglitazona y la pioglitazona (tiazolidinedionas), pertenecen a la categoría C para su uso en el embarazo y probable- mente no son medicamentos útiles en la prevención del aborto en pacientes con SOP. Las tiazolidinedionas en estudios en roedores mostraron que atraviesan la placenta y pueden producir disminución del peso fetal y pérdida posimplantación. ${ }^{26}$ No hay estudios controlados en mujeres embarazadas, como tampoco hay estudios en embarazadas con el D- Chiroinositol.

Otras medidas terapéuticas que contribuyen a la disminución de los niveles de insulina, tales como los cambios en el estilo de vida (ejercicio, disminución del peso), mejoran el ambiente hormonal y metabólico de las pacientes con $\mathrm{SOP}^{27}$ y por lo tanto, se podría especular que mejorarían la receptividad endometrial, la implantación y eventualmente, el riesgo de aborto.

\section{CONCLUISIONES}

Se acepta que las mujeres con SOP tienen un mayor riesgo de aborto. Los factores etiológicos descritos para explicar este hecho son: concentraciones elevadas de $\mathrm{LH}$, concentraciones elevadas de insulina y concentraciones elevadas de andrógenos. El reflejo de estos fenómenos a nivel endometrial demostrado en una alteración en los niveles de los receptores de andrógenos, receptores de estrógenos, integrina $\alpha_{v} \beta 3$, glicodelina, IGFs, IGFBP-1, entre otros, podría promover la inadecuada implantación y eventualmente aumentar el riesgo de aborto. Ya en el pasado otras enfermedades como la preeclampsia, se han asociado a anormalidades en los procesos de implantación. ${ }^{28}$

Aunque los fenómenos antes mencionados podrían explicar el aumento de la tasa de aborto en las pacientes con SOP, algunos autores como el doctor Norman, ${ }^{26}$ piensan que aun no está plenamente confirmada la asociación entre el aborto y el SOP, y sugiere que esta asociación es producida más por factores independientes como la obesidad y la resistencia a la insulina.

Hay algunos estudios observacionales que sugieren que la metformina disminuye el riesgo de aborto en pacientes con SOP, asociado esto a una 
mejor sensibilidad a la insulina, un aumento en los niveles de glicodelina y de IGFBP, y una disminución de los niveles de $\mathrm{LH}{ }^{26,28}$ Con frecuencia tendremos pacientes con SOP que se embarazan recibiendo metformina. Es probable que mantener su uso durante el primer trimestre de embarazo pueda disminuir el riesgo de aborto. Se necesitan estudios aleatorizados y prospectivos que confirmen la utilidad de la metformina para este propósito.

\section{REFERENCIAS}

1. Barbieri RL. Metformin for the treatment of polycystic ovary syndrome. Obstet Gynecol 2003;101:785-93.

2. Lathi RB, Swierz L, Basina M, Giudice LC. The endometrium in polycystic ovary syndrome. Curr Opin Endocrinol Diabetes 2002;9:480-5.

3. Genazzani A, Battaglia C, Malavasi B, Strucchi C, Tortolani F, Gamba O. Metformin administration modulates and restores luteinizing hormone spontaneous episodic secretion and ovarian function in nonobese patients with polycystic ovary syndrome. Fertil Steril 2004:81:114-9.

4. Glueck CJ, Phillips H, Cameron D, Sieve-Smith L, Wang P. Continuing metformin throughout pregnancy in women with polycystic ovary syndrome appears to safely reduce first-trimester spontaneous abortion: a pilot study. Fertil Steril 2001:75:46-52.

5. The Rotterdam ESHRE-ASRM-sponsored PCOS Consensus Workshop Group. Revised 2003 consensus on diagnostic criteria and long-term health risks related to polycystic ovary syndrome (PCOS). Hum Reprod 2004;19:41-7.

6. Ehrman DA. Polycystic ovary syndrome. N Engl J Med 2005;352:1223-36.

7. Rebar R, Judd HL, Yen SS, Rakoff J, Vandenberg G, and Naftolin F. Characterization of the Inappropriate gonadotropin secretion in polycystic ovary syndrome. J Clin Invest 1976;57:1320-9.

8. DeUgarte CM, Bartolucci AA, Azziz R. Prevalence of insulin resistance in the polycystic ovary syndrome using the homeostasis model assessment. Fertil Steril 2005;83:1454-60.

9. Goodarzi MO, Korenman SG. The importance of insulin resistance in polycystic ovary syndrome. Fertil Steril 2003;80:255-8.
10. Cheviakoff S, Carmona S, Lahsen R. Estudios de variables clínicas y metabólicas en mujeres con hiperandrogenismo. Rev Chil Obstet Ginecol 2004;69:39-43.

11. Giudice LC. Potential biochemical markers of uterine receptivity. Hum Reprod 1999;14 Suppl 2:3-16.

12. Daftary GS, Taylor HS. Molecular markers of implantation: clinical implications. Curr Opin Obstet Gynecol 2001;13:269-74.

13. Coetzee EJ, Jackson WP. Pregnancy in established non-insulin-dependent diabetics. A five-and-a-half year study at Groote Schuur Hospital. S Afr Med J 1980;58:795-802.

14. Clifford K, Rai R, Watson H, Franks S, Regan L. Does suppressing luteinising hormone secretion reduce the miscarriage rate? Results of a randomised controlled trial. BMJ 1996;312:1508-11.

15. Regan L, Owen EJ, Jacobs HS. Hypersecretion of luteinising hormone, infertility, and miscarriage. Lancet 1990;336:1141-4..

16. Homburg R, Armar NA, Eshel A, Adams J, Jacobs HS. Influence of serum luteinising hormone concentrations on ovulation, conception, and early pregnancy loss in polycystic ovary syndrome. BMJ 1988;297:1024-6.

17. Fedorcsak P, Storeng R, Dale PO, Tanbo T, Abyholm T. Obesity is a risk factor for early pregnancy loss after IVF or ICSI. Acta Obstet Gynecol Scand 2000;79:43-8.

18. Glueck CJ, Wang P, Fontaine RN, Sieve-Smith L, Tracy T, Moore SK. Plasminogen activator inhibitor activity: an independent risk factor for the high miscarriage rate during pregnancy in women with polycystic ovary syndrome. Metabolism 1999;48:1589-95.

19. Gris JC, Ripart-Neveu S, Maugard C, Tailland ML, Brun S, Courtieu C, et al. Retrospective evaluation of the prevalence of haemostasis abnormalities in unexplained primary early recurrent miscarriages. The Nimes Obstetricians and Haematologists (NOHA) study. Thromb Haemost 1997;77:1096-103.

20. Checa MA, Requena A, Salvador C, Tur R, Espinos JJ, et al. Reproductive Endocrinology Interest Group of the Spanish Society of Fertility. Insulin-sensitizing agents: use in pregnancy and as therapy in polycystic ovary syndrome. Hum Reprod Update 2005:11:375-90.

21. Lord J, Wilkin T. Metformin in polycystic ovary syndrome. Curr Opin Obstet Gynecol 2004;16:481-6. 
22. Vanky E, Salvesen KA, Heimstad R, Fougner KJ, Romundstad P, Carlsen SM. Metformin reduces pregnancy complications without affecting androgen levels in pregnant polycystic ovary syndrome women: results of a randomized study. Hum Reprod 2004;19:1734-40.

23. Reis RM, Angelo A, Romao G, Santana L, Moura M, Ferriani RA. Can polycystic ovary syndrome interfere with the outcome of in vitro fertilization? Rev Bras Ginecol Obstet 2004;26:727-33.

24. Glueck CJ, Wang P, Goldenberg N, Sieve-Smith L. Pregnancy outcomes among women with polycystic ovary syndrome treated with metformin. Hum Reprod 2002;17:2858-64.

25. Glueck CJ, Goldenberg N, Pranikoff J, Loftspring M, Sieve L, Wang P. Height, weight, and motor- social development during the first 18 months of life in 126 infants born to 109 mothers with polycystic ovary syndrome who conceived on and continued metformin through pregnancy. Hum Reprod 2004;19:1323-30

26. Norman RJ, Wang JX, Hague W. Should we continue or stop insulin sensitizing drugs during pregnancy? Curr Opin Obstet Gynecol 2004;16: 245-50.

27. Lord JM, Flight IHK, Norman RJ. Insulin-sensitising drugs (metformin, troglitazone, rosiglitazone, pioglitazone, D-chiro-inositol) for polycystic ovary syndrome (Cochrane Review). En: The Cochrane Library, Issue 3, 2003. Oxford: Update Software.

28. Irwin JC, Suen LF, Martina NA, Mark SP, Giudice LC. Rol of the IGF system in trophoblast invasion and pre-eclampsia. Hum Reprod 1999;14 Suppl 2:90-6.

Conflicto de intereses: ninguno declarado. 